\title{
Malignant Pleural Mesothelioma Epidemiology in the United States From 2000 to 2016
}

\author{
Akesh Thomas $^{1}$, Sajin Karakattu ${ }^{1}$, Jeanette Cagle ${ }^{1}$, Girendra Hoskere ${ }^{2}$ \\ 1. Internal Medicine, East Tennessee State University Quillen College of Medicine, Johnson City, USA 2. Pulmonary and \\ Critical Care Medicine, East Tennessee State University Quillen College of Medicine, Johnson City, USA
}

Corresponding author: Akesh Thomas, thomasa16@etsu.edu

\section{Abstract \\ Introduction}

Pleural mesothelioma constitutes about $80 \%$ of all mesotheliomas. The peak incidence of malignant mesothelioma estimated using the cancer registries was in early 1990 to 2000 in the United States. The disease is primarily associated with asbestos exposure. The latency period between asbestos exposure and the development of malignant pleural mesothelioma (MPM) can range anywhere from 15 to 60 years. Asbestos exposure was peaked during the industrial revolution and World War II due to military and shipyard exposures. It is often difficult for the pathologist to distinguish different histological subtypes; due to the disease's rarity and the inadequate tissue sample obtained. There is no available data on the difference in epidemiology of different subtypes of MPM. Surveillance Epidemiology and End Results (SEER), cancer incidence data include population-based registries covering approximately $34.6 \%$ of the U.S. population. Here in our study, we analyze malignant pleural mesothelioma epidemiology in the United States, emphasizing different histological subtypes.

\section{Methods}

SEER data from 2000 to 2016 was used in our study. The primary site of cancer is selected as pleura, and malignant behavior only is selected as the filter. Data were analyzed using the SEER stat program. Overall epidemiology of MPM and epidemiology of epithelioid, fibrous, and biphasic histological subtypes were analyzed separately. We used annual percentage change (APC) to evaluate the trend in the epidemiology of MPM.

Review began 03/28/2021 Review ended 04/19/2021 Published 04/21/2021

(c) Copyright 2021

Thomas et al. This is an open access article distributed under the terms of the Creative Commons Attribution License CC-BY 4.0., which permits unrestricted use, distribution, and reproduction in any medium, provided the original author and source are credited.

\section{Results summary}

A total of 11,857 cases of MPM were included in the primary cohort from the SEER 18 registry from 2000 to 2016. The total prevalence of MPM was highest in 2009 and was lowest in 2016. The APC in MPM incidence during this period is -2.0 . After removing 5,989 cases with non-specified histology during the same period, the APC for each histological type is -0.7 for fibrous type, 1.8 for epithelioid type, and 2.9 for biphasic type. Out of 17 regional registries included in the study, the greatest statistically significant change in APC was seen in the Hawaiian registry -4.1. In contrast, the lowest statistically significant difference was seen in Seattle (Puget Sound) registry -1.7. The APC in the incidence of MPM among males during the study period was -2.4 while that of females was -0.9 . The Iowa registry showed a statistically significant increase in APC of the epithelioid malignant mesothelioma with a statistically insignificant reduction in the overall MPM APC.

\section{Conclusion}

The overall incidence of MPM in the United States is declining, while the data showed an increase in the incidence of epithelioid and biphasic histological subtypes. The authors believe that these conflicting results can be attributed to improved histological diagnosis and improved biopsy techniques.

Categories: Oncology, Pulmonology, Public Health

Keywords: malignant pleural mesothelioma, asbestos, mesothelioma, national cancer database and seer analyses

\section{Introduction}

Mesothelioma is a neoplasm originating from the body's mesothelial surfaces, with about $80 \%$ of cases being pleural in origin. The United States (U.S.) cancer statistics division of the center for disease control (CDC) estimates the five-year prevalence of mesothelioma to be about 4,562 in the United States [1]. The peak incidence of malignant mesothelioma estimated using the cancer registries was in early 1990 to 2000 in the United States [2], while the peak incidence in England was around the year 2015 [3]. The disease is mostly associated with asbestos exposure; one case per million of the general population is the incidence of nonasbestos-related mesothelioma [4]. Radiation exposure is a significant risk factor in non-asbestos-related cases [5]. The latency period between asbestos exposure and the development of malignant pleural 
mesothelioma (MPM) can range anywhere from 15 to 60 years [6]. Three different histological subtypes of MPM are typically identified; epithelioid, sarcomatoid, and biphasic [7]. It is often difficult for the pathologist to distinguish different histological subtypes due to the disease's rarity and the inadequate tissue sample obtained. There is no available data on the difference in epidemiology of different histological subtypes of MPM. But, studies suggest that the epithelioid subtype is associated with better survival than the other subtypes $[8,9]$.

Asbestos exposure peaked during the industrial revolution and World War II due to military and shipyard exposures [10]. The mesothelioma hot spots in the Unites States can be traced to communities that lived near industries that used asbestos in abundance, like Manville in New Jersey and Libby in Montana. On the other hand, California has the largest natural asbestos deposit, increasing MPM incidence in the state. Asbestos is the generic name for a group of naturally occurring minerals that contain silicate tetrahedron (SiO4). The arrangement and number of tetrahedra determine the classification of the mineral. Six arrangements are classified as asbestos: chrysotile, actinolite, amosite, anthophyllite, crocidolite, and tremolite. Each of these arrangements has varying chemical and physical properties, but all share carcinogenic risks. Exposure through inhalation and ingestion must occur for asbestos to cause cancer. Other methods of exposure, such as dermatologic contact, are not associated with any carcinogenic risk. Exposures to crocidolite, amosite, and chrysotile are most commonly associated with pleural mesothelioma development [11]. Asbestos in all forms is also associated with the development of pharynx, stomach, and colorectal cancers [12]. The determinants of asbestos toxicity are fiber size, bio persistence, chemical composition, and particle surface characteristics [13]. The proposed mechanisms by which asbestos causes carcinogenesis are direct interaction with cellular chromosomes, generation of reactive oxygen species, and inflammation [14]. The occupational asbestos exposure has been significantly reduced since the 1970s, leading to a steady decline in MPM incidence after the year 2000. The world trade center disaster in 2001caused significant asbestos particle exposure among people living in New York City and those involved in rescue work [15], which can lead to MPM development decades later.

Surveillance, Epidemiology, and End Results (SEER) cancer incidence data include population-based registries covering approximately $34.6 \%$ of the U.S. population. Here in our study, we analyze malignant pleural mesothelioma epidemiology in the United States, emphasizing different histological subtypes.

\section{Materials And Methods}

SEER data from 2000 to 2016 was used in our study. Data included 18 regional registries in SEER. Cancer's primary site was selected as pleura, and malignant behavior only was chosen as the filter in SEER software. We excluded age zero and unknown age from the data. For the histological classification, we excluded the unknown histological type. Data were analyzed using the SEER stat program [16]. Overall epidemiology of MPM and epidemiology of epithelioid, fibrous, and biphasic histological subtypes were studied separately. Data were analyzed independently for gender, ethnicity, age groups of 45-49, 50-54, 55-59, 60-64, 65-69, 70$74,75-79$, and the 17 different regions. We used annual percentage change (APC) to evaluate the trend in the epidemiology of MPM. The APC was calculated using the incidence rate per 100,000 of the U.S. population age-adjusted to the 2000 U.S. standard population (19 age groups - Census P25-1130). The percentage change is calculated using one year for each endpoint. A 95\% confidence interval is included with all the calculated results for assessing the significance.

\section{Results}

A total of 11,857 cases of MPM were included in the primary cohort from the SEER 18 registry from 2000 to 2016. The total prevalence of MPM was highest in 2000 and was lowest in 2016. The overall prevalence of MPM decreased during the study period, while the epithelioid MPM prevalence increased during the same period (Figure 1). The overall annual percentage change (APC) in MPM incidence during this period is -2.0, $95 \%$ CI $[-2.4,-1.5]$. The APC for each histological type after removing 5,989 cases with non-specified histology during the same period was $-0.7[-3.1,1.7]$ for fibrous type, $1.8[1.2,2.3]$ for epithelioid type, and 2.9 $[0.8,5.1]$ for biphasic type. Out of 17 regional registries included in the study, the greatest statistically significant change in APC was seen in the Hawaiian registry $-4.1[-7.6,-0.4]$ while the lowest statistically significant difference was seen in Seattle (Puget Sound) registry -1.7 [-2.7, -0.6]. A significant change in APC was seen in San Francisco-Oakland, Connecticut, Seattle, Los Angeles, California, and New Jersey registries. In contrast, Detroit(metropolitan), Iowa, New Mexico, Utah, Atlanta, San Jose-Monterey, Kentucky, Louisiana, and Greater Georgia registries did not show a significant change in APC (Figure 2). Detroit metropolitan region, Seattle (Puget Sound), Louisiana, and New Jersey, registries showed a statistically significant increase in the epithelioid variant of MPM while the overall APC for MPM in the region still showed a statistically significant decrease during the study period. Contrary to this, the Iowa registry showed a statistically significant increase in APC of the epithelioid malignant mesothelioma with a statistically insignificant reduction in the APC overall for MPM. Statistical calculations were not possible in region-wise APC for fibrous and biphasic MPM in most regions due to the inadequate number of patients. The APC in the incidence of MPM among males during the study period was $-2.4[-3,-1.8]$ while that of females was $-0.9[-$ $1.5,-0.2]$. Of all the age groups from 45 to 85 (in multiples of 5), the APC for overall malignant mesothelioma showed a statistically significant decrease in all the groups. Fibrous MPM showed a statistically significant reduction in the age group of 60-64 only. Epithelioid MPM showed a statistically significant reduction in 


\section{Cureus}

APC among age groups 50-54 and 55-59, while it showed a statistically significant increase in age groups 7074,75-79, and 80-84. Biphasic MPM showed a statistically significant increase in the age group 65-69 only (Figure 3). Blacks, whites, and Asian/pacific islanders showed a statistically significant decrease in the APC of overall MPM. While whites showed a statistically significant increase in APC for both epithelioid and biphasic MPM, all the other racial subclasses were either statistically insignificant or unable to calculate. (For detailed data, see Tables $1-3$ in the appendix).

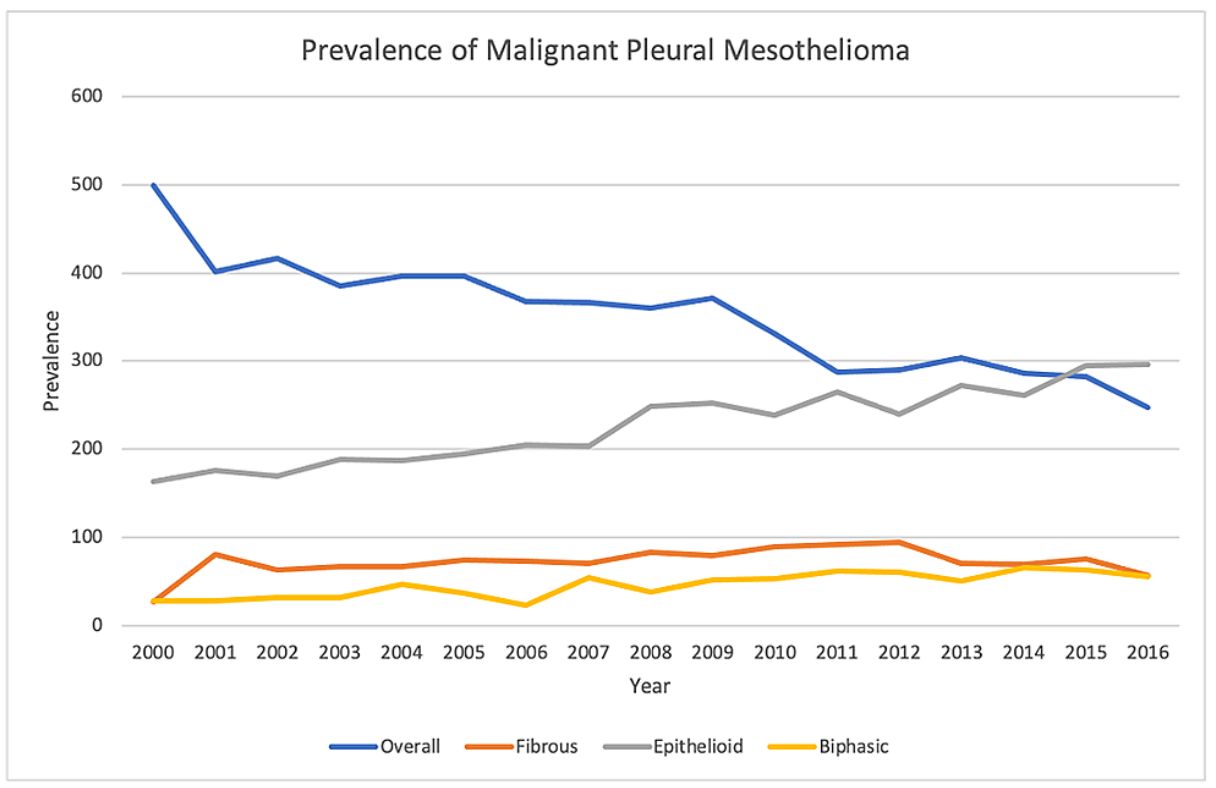

FIGURE 1: Prevalence of malignant pleural mesothelioma.

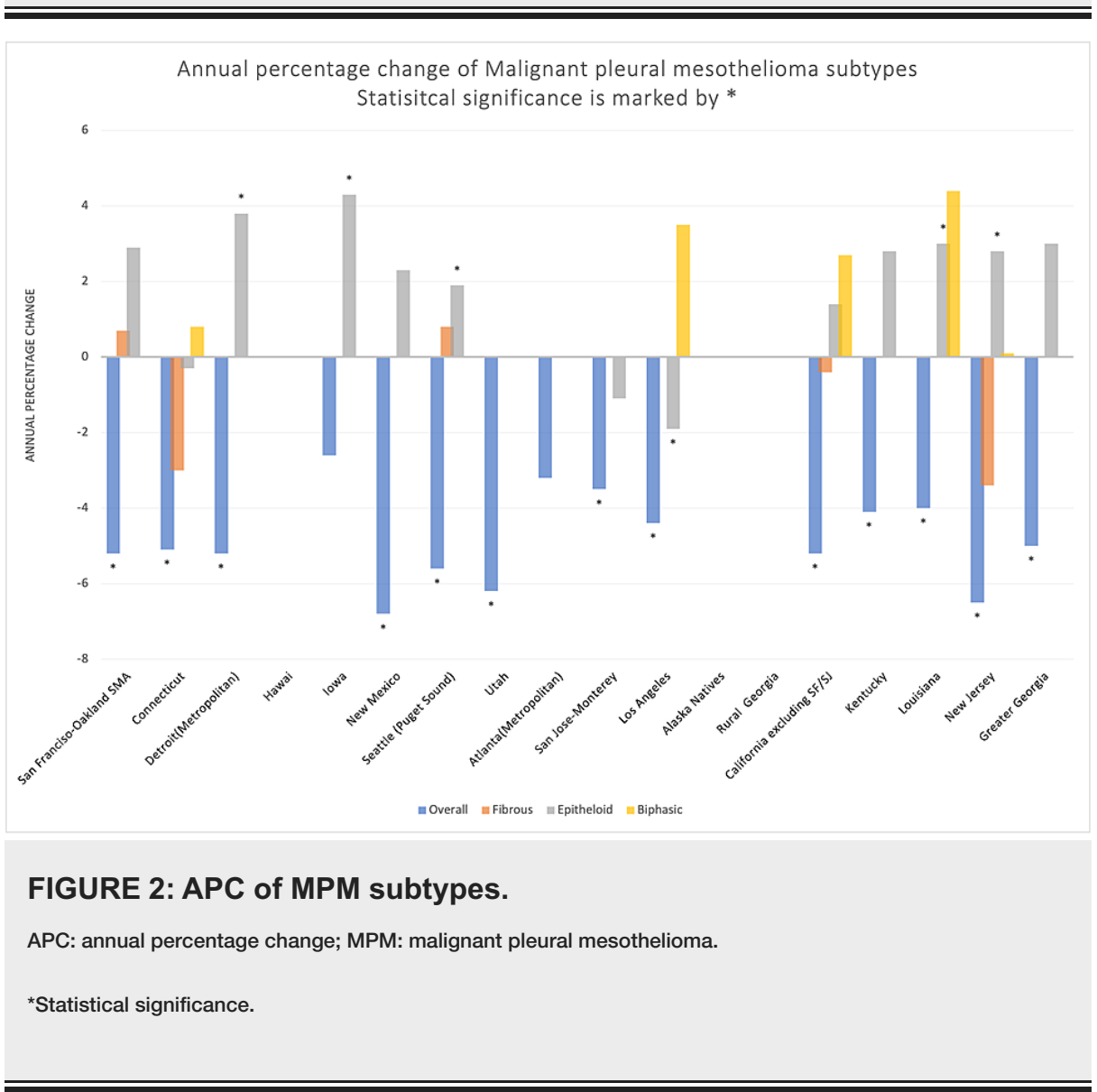




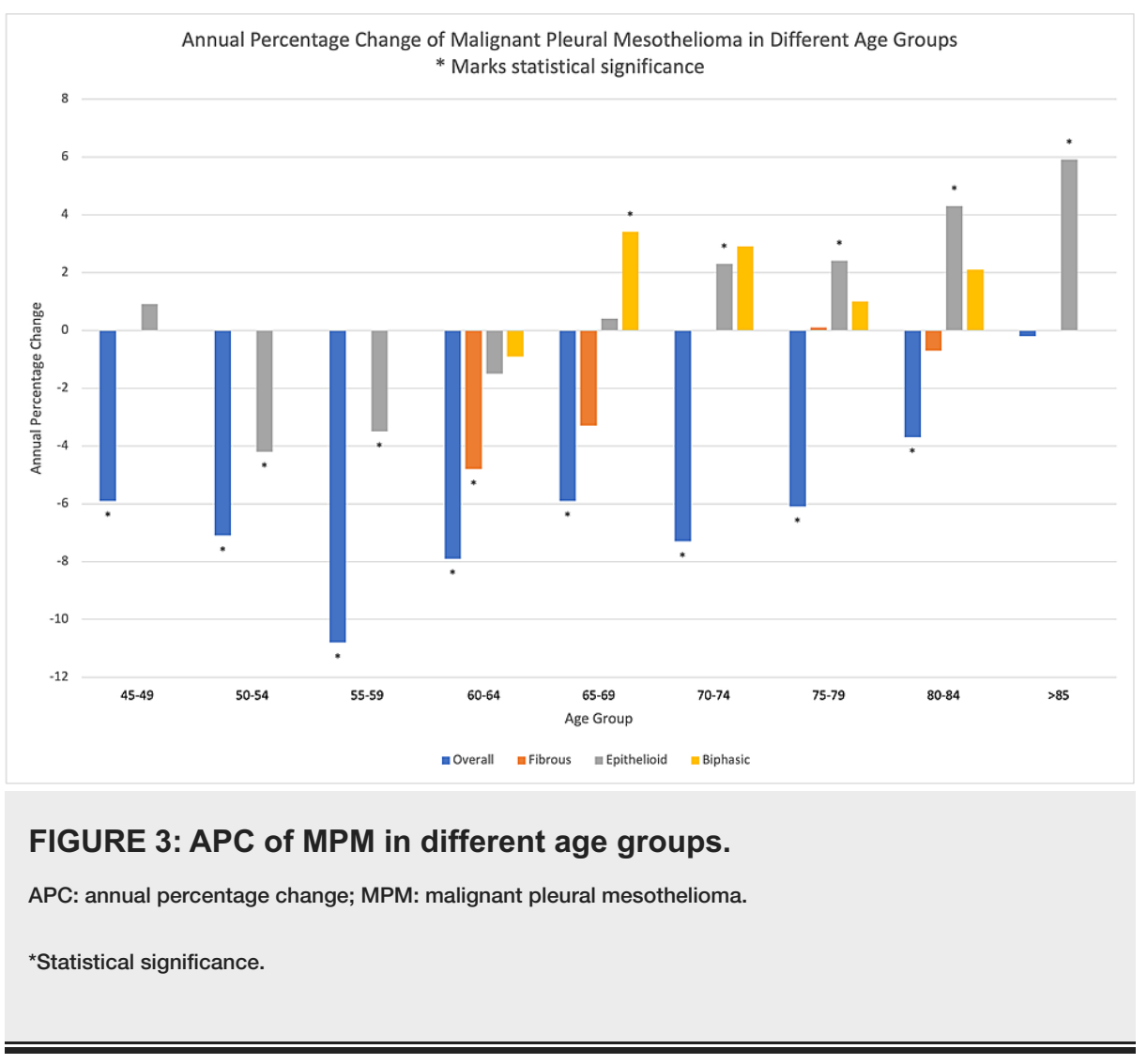

\section{Discussion}

The analysis of the MPM epidemiology clearly shows a decline in the total prevalence and overall incidence of MPM in the United States. Despite this, there is an increase in the prevalence of epithelioid and biphasic MPM subtypes with a positive APC during the study period. The authors believe this paradoxical statistic results from increased diagnostic accuracy of MPM histological subtypes during the study period. Increased awareness of the histological variants, availability of better tissue samples with increased access to better techniques like video-assisted thoracoscopy, and improved pathological diagnostic techniques might have helped. The challenges that pathologists faced in making an accurate diagnosis of mesothelioma is well reported in the literature [17]. Magner and McDonald in 1972 suggested the association of histological types of MPM differently to asbestos exposure (with mixed cell type more likely associated with asbestos exposure) [18], but this hypothesis was later disproved on further analysis [19]. Most regional MPM statistics followed the national trend as expected, with a negative APC for overall MPM incidence with either positive or negative APC for the epithelioid subtype. An exception is the Iowa registry, which showed a statistically significant positive APC for the incidence of epithelioid MPM with a statistically non-significant negative APC for overall MPM incidence. Whether the Iowa registry is of concern needs further detailed investigation. Among all age groups from 45 to 85 , there is a significant decline in the overall incidence of MPM. A significant increase in the epithelioid MPM is seen in persons older than 70. During the study period, those older than 70 are members of the population that lived through the second world war and the industrial revolution, with obviously more asbestos exposure than the younger people during the study period. Is this a mere coincidence or a suggestion of a correlation between asbestos exposure and epithelioid type MPM (than other histological types) needs further detailed analysis. Besides, there are suggestions that the mesothelioma in young people (age < 40) may be less related to asbestos exposure than those seen in the older population [20].

The accurate diagnosis of MPM histological subtype needs special training for the pathologist [21]. The most common subtype epithelioid MPM consists of a heterogeneous group of histopathology, including solid, tubulopapillary, trabecular, micropapillary, deciduoid, and pleomorphic [22]. Even within these variants of epithelioid subtype, the prognosis varies significantly [23,24], which signifies the importance of making an accurate histological diagnosis important. We recommend that all the MPM diagnoses should be confirmed for the histology in a center with expertise in the pathological diagnosis of MPM.

The authors acknowledge that the study is limited by the basic characteristics of the registry itself. The study's retrospective nature and the lack of information on whether the histopathological diagnosis was made in an expert center are limitations of the study. Deriving a conclusion on the epidemiology of the different histological types of MPM using the registry data can be potentially erroneous due to numerous biases, including selection bias and reporting bias. The lack of data from some of the states with a high incidence of MPM, like Pennsylvania, Florida, and Texas, is another limitation of this study. 


\section{Cureus}

\section{Conclusions}

The overall incidence of MPM in the United States is declining, while the data showed an increase in the incidence of epithelioid and biphasic histological subtypes. The authors believe that these conflicting results can be attributed to the improved histological diagnosis and improved biopsy techniques, including video-assisted thoracoscopy. Iowa registry showed a significant increase in epithelioid MPM without a significant decline in overall MPM incidence. There is more decline in MPM incidence in males than females, while the incidence of MPM in males still remains higher than in females.

\section{Appendices}

\begin{tabular}{|c|c|c|c|c|}
\hline Year & Mesothelioma total & Fibrous mesothelioma & Epithelioid mesothelioma & Biphasic mesothelioma \\
\hline 2000 & 499 & 26 & 163 & 28 \\
\hline 2001 & 402 & 81 & 176 & 28 \\
\hline 2002 & 417 & 63 & 170 & 32 \\
\hline 2003 & 385 & 67 & 188 & 32 \\
\hline 2004 & 396 & 67 & 187 & 46 \\
\hline 2005 & 396 & 74 & 195 & 36 \\
\hline 2006 & 368 & 73 & 205 & 23 \\
\hline 2007 & 366 & 71 & 203 & 54 \\
\hline 2008 & 360 & 83 & 249 & 38 \\
\hline 2009 & 372 & 79 & 252 & 52 \\
\hline 2010 & 331 & 89 & 239 & 53 \\
\hline 2011 & 288 & 92 & 265 & 62 \\
\hline 2012 & 290 & 94 & 240 & 61 \\
\hline 2013 & 304 & 71 & 272 & 51 \\
\hline 2014 & 286 & 69 & 261 & 66 \\
\hline 2015 & 282 & 76 & 295 & 63 \\
\hline 2016 & 247 & 57 & 296 & 55 \\
\hline
\end{tabular}

TABLE 1: Number of newly diagnosed malignant pleural mesothelioma cases per year. 


\section{Cureus}

\begin{tabular}{|c|c|c|c|}
\hline Region & Rate/trend & Lower $\mathrm{Cl}$ & Upper CI \\
\hline San Francisco-Oakland SMSA - 2000+ & $-2.1^{*}$ & -3.8 & -0.4 \\
\hline Connecticut - 2000+ & $-2.5^{\star}$ & -4.6 & -0.3 \\
\hline Detroit (Metropolitan) - 2000+ & -0.9 & -3 & 1.3 \\
\hline Hawaii - 2000+ & $-4.1^{*}$ & -7.6 & -0.4 \\
\hline lowa - 2000+ & 1.4 & -0.9 & 3.7 \\
\hline New Mexico - 2000+ & -2.3 & -5.3 & 0.8 \\
\hline Seattle (Puget Sound) - 2000+ & $-1.7^{\star}$ & -2.7 & -0.6 \\
\hline Utah - 2000+ & -2.3 & -5 & 0.4 \\
\hline Atlanta (Metropolitan) - 2000+ & -0.8 & -3.2 & 1.7 \\
\hline San Jose-Monterey - 2000+ & -1.5 & -3.7 & 0.8 \\
\hline Los Angeles - 2000+ & $-2.6^{*}$ & -3.8 & -1.5 \\
\hline Rural Georgia - 2000+ & $\sim$ & $\sim$ & $\sim$ \\
\hline California excluding SF/SJM/LA - 2000+ & $-2.2^{*}$ & -3.4 & -1.1 \\
\hline Kentucky - 2000+ & -0.4 & -2 & 1.2 \\
\hline Louisiana - 2000+ & -0.7 & -1.8 & 0.4 \\
\hline New Jersey - 2000+ & $-2.8^{*}$ & -3.8 & -1.9 \\
\hline Greater Georgia - 2000+ & -1.7 & -3.7 & 0.3 \\
\hline
\end{tabular}

\section{TABLE 2: Overall MPM annual percentage change in different registries.}

*Statistically significant. Cannot be calculated.

MPM: malignant pleural mesothelioma. 


\section{Cureus}

\begin{tabular}{|c|c|c|c|c|}
\hline Region & Fibrous & Epithelioid & Biphasic & Total \\
\hline San Francisco-Oakland SMSA - 2000+ & 0.7 & 2.9 & $\sim$ & $-5.2^{\star}$ \\
\hline Connecticut - 2000+ & -3 & -0.3 & 0.8 & $-5.1^{*}$ \\
\hline Detroit (Metropolitan) - 2000+ & $\sim$ & $3.8^{*}$ & $\sim$ & $-5.2^{*}$ \\
\hline Hawaii - 2000+ & $\sim$ & $\sim$ & $\sim$ & $\sim$ \\
\hline lowa - 2000+ & $\sim$ & 4.3 & $\sim$ & -2.6 \\
\hline New Mexico - 2000+ & $\sim$ & 2.3 & $\sim$ & $-6.8^{*}$ \\
\hline Seattle (Puget Sound) - 2000+ & 0.8 & $1.9^{*}$ & $\sim$ & $-5.6^{*}$ \\
\hline Utah - 2000+ & $\sim$ & $\sim$ & $\sim$ & $-6.2^{*}$ \\
\hline Atlanta (Metropolitan) - 2000+ & $\sim$ & $\sim$ & $\sim$ & -3.2 \\
\hline San Jose-Monterey - 2000+ & $\sim$ & -1.1 & $\sim$ & $-3.5^{\star}$ \\
\hline Los Angeles - 2000+ & $\sim$ & $-1.9^{*}$ & 3.5 & $-4.4^{\star}$ \\
\hline Rural Georgia - 2000+ & $\sim$ & $\sim$ & $\sim$ & $\sim$ \\
\hline California excluding SF/SJM/LA - 2000+ & -0.4 & 1.4 & 2.7 & $-5.2^{\star}$ \\
\hline Kentucky - 2000+ & $\sim$ & 2.8 & $\sim$ & $-4.1^{\star}$ \\
\hline Louisiana - 2000+ & $\sim$ & $3.0^{*}$ & 4.4 & $-4.0^{*}$ \\
\hline New Jersey - 2000+ & -3.4 & $2.8^{*}$ & 0.1 & $-6.5^{\star}$ \\
\hline Greater Georgia - 2000+ & $\sim$ & 3 & $\sim$ & -5 \\
\hline
\end{tabular}

\section{TABLE 3: Annual percentage change of histological subtypes of MPM.}

*Statistically significant. Cannot be calculated.

MPM: malignant pleural mesothelioma.

\section{Additional Information}

\section{Disclosures}

Human subjects: All authors have confirmed that this study did not involve human participants or tissue. Animal subjects: All authors have confirmed that this study did not involve animal subjects or tissue. Conflicts of interest: In compliance with the ICMJE uniform disclosure form, all authors declare the following: Payment/services info: All authors have declared that no financial support was received from any organization for the submitted work. Financial relationships: All authors have declared that they have no financial relationships at present or within the previous three years with any organizations that might have an interest in the submitted work. Other relationships: All authors have declared that there are no other relationships or activities that could appear to have influenced the submitted work.

\section{References}

1. U.S. Cancer Statistics Working Group. U.S. Cancer Statistics Data Visualizations Tool, based on 2019 submission data (1999-2017): U.S. Department of Health and Human Services, Centers for Disease Control and Prevention and National Cancer Institute. (2020). http://www.cdc.gov/cancer/dataviz.

2. Price B: Analysis of current trends in United States mesothelioma incidence . Am J Epidemiol. 1997, 145:211-8. 10.1093/oxfordjournals.aje.a009093

3. Hodgson JT, McElvenny DM, Darnton AJ, Price MJ, Peto J: The expected burden of mesothelioma mortality in Great Britain from 2002 to 2050. Br J Cancer. 2005, 92:587-93. 10.1038/sj.bjc.6602307

4. Price B: Analysis of current trends in United States mesothelioma incidence . Am J Epidemiol. 1997, 145:211-8. 10.1093/oxfordjournals.aje.a009093

5. Teta MJ, Lau E, Sceurman BK, Wagner ME: Therapeutic radiation for lymphoma: risk of malignant mesothelioma. Cancer. 2007, 109:1432-8. 10.1002/cncr.22526

6. Bianchi C, Giarelli L, Grandi G, Brollo A, Ramani L, Zuch C: Latency periods in asbestos-related mesothelioma of the pleura. Eur J Cancer Prev. 1997, 6:162-6.

7. Ascoli V, Minelli G, Cozzi I, Romeo E, Carnovale Scalzo C, Ancona L, Forastiere F: Pathology reporting of 
malignant pleural mesothelioma first diagnosis: a population-based approach. Pathol Res Pract. 2016, 212:886-92. 10.1016/j.prp.2016.07.010

8. Meyerhoff RR, Yang CF, Speicher PJ, et al.: Impact of mesothelioma histologic subtype on outcomes in the Surveillance, Epidemiology, and End Results database. J Surg Res. 2015, 196:23-32. 10.1016/j.jss.2015.01.043

9. Rusch VW, Giroux D, Kennedy C, et al.: Initial analysis of the international association for the study of lung cancer mesothelioma database. J Thorac Oncol. 2012, 7:1631-9. 10.1097/JTO.0b013e31826915f1

10. Ruffie PA: Pleural mesothelioma. Curr Opin Oncol. 1991, 3:328-34. 10.1097/00001622-199104000-00014

11. IARC Working Group on the Evaluation of Carcinogenic Risks to Humans: Arsenic, metals, fibres, and dusts . 2012, 100:

12. Straif K, Benbrahim-Tallaa L, Baan R, Grosse Y, et al. WHO International Agency for Research on Cancer Monograph Working Group: A review of human carcinogens--Part C: metals, arsenic, dusts, and fibres . Lancet Oncol. 2009, 10:453-4. 10.1016/s1470-2045(09)70134-2

13. Klebe S, Henderson DW: The molecular pathogenesis of asbestos-related disorders. Asbestos: risk assessment, epidemiology, and health effects, 2nd ed. Dodson RF, Hammar SP (ed): CRC Press, Taylor \& Francis Group, Bacon Raton, FL; 2011.

14. Brody AR: Asbestos and lung disease. Am J Respir Cell Mol Biol. 2010, 42:131-2. 10.1165/rcmb.2010-2002ED

15. Landrigan PI: The WTC disaster: Landrigan's response . Environ Health Perspect. 2004, 112:A607.

16. Surveillance Research Program: National Cancer Institute SEER*Stat software (seer.cancer.gov/seerstat) version 8.3.8

17. McDonald AD, Magner D, Eyssen G: Primary malignant mesothelial tumors in Canada, 1960-1968. A pathologic review by the Mesothelioma Panel of the Canadian Tumor Reference Centre. Cancer. 1973, 31:869-76. 10.1002/1097-0142(197304)31:4<869::aid-cncr2820310416>3.0.co;2-s

18. Magner D, McDonald AD: Malignant mesothelial tumors - histologic type and asbestos exposure. N Engl J Med. 1972, 287:570-1.

19. Wright WE, Sherwin RP: Histological types of malignant mesothelioma and asbestos exposure . Br J Ind Med. 1984, 41:514-7. 10.1136/oem.41.4.514

20. Lemen RA: Mesothelioma from asbestos exposures: epidemiologic patterns and impact in the United States . J Toxicol Environ Health B Crit Rev. 2016, 19:250-65. 10.1080/10937404.2016.1195323

21. Brcic L, Vlacic G, Quehenberger F, Kern I: Reproducibility of malignant pleural mesothelioma histopathologic subtyping. Arch Pathol Lab Med. 2018, 142:747-52. 10.5858/arpa.2017-0295-OA

22. Galateau-Salle F, Churg A, Roggli V, et al.: The 2015 World Health Organization Classification of Tumors of the Pleura: Advances since the 2004 Classification. J Thorac Oncol. 2016, 11:142-154. 10.1016/j.jtho.2015.11.005

23. Alchami FS, Attanoos RL, Bamber AR: Myxoid variant epithelioid pleural mesothelioma defines a favourable prognosis group: an analysis of 191 patients with pleural malignant mesothelioma. J Clin Pathol. 2017, 70:179-82. 10.1136/jclinpath-2016-203993

24. Ordóñez NG: Pleomorphic mesothelioma: report of 10 cases. Mod Pathol. 2012, 25:1011-22. 10.1038/modpathol.2012.39 